\title{
A990, Rat Strain
}

National Cancer Institute

\section{Source}

National Cancer Institute. A990, Rat Strain. NCI Thesaurus. Code C14389.

Resistant to Cysticercus. Susceptible to estrogen-induced mammary and adrenal tumors. Good reproduction. High open-field defecation (2/12, males, 3/12 females) and low ambulation (11/12), Harrington (1972). Low wheel activity (12/12) (Harrington 1971b). Agg ressive towards handlers (Harrington 1981). 